\title{
Bayesian Estimation of the Exponential Parameter under a Multiply Type-II Censoring Scheme
}

\author{
Umesh Singh $^{1}$ and Anil Kumar ${ }^{2}$ \\ ${ }^{1}$ Banaras Hindu University, Varanasi, India \\ ${ }^{2}$ Mahatma Gandhi Kashi Vidyapith, Varanasi, India
}

\begin{abstract}
This paper provides the estimation of the scale parameter of the exponential distribution under multiply type-II censoring. Using generalized non-informative prior and natural conjugate prior, Bayes estimator and approximate Bayes estimators of the scale parameter have been obtained under square error loss function. The proposed Bayes estimators and approximate Bayes estimators are compared with the estimators proposed by Singh et al. (2005) and Balasubramanian and Balakrishnan (1992) on the basis of their simulated risks under square error loss function of 1000 randomly generated Monte Carlo samples.

Zusammenfassung: Dieser Aufsatz bietet Schätzer des Skalenparameters der Exponentialverteilung unter multipler Type-II Zensierung an. Unter Verwendung eines generalisierten nicht-informativen wie auch eines natürlichen konjugierten Priors erhält man Bayes- und approximative Bayes-Schätzer des Skalenparameters unter quadratischer Verlustfunktion. Die vorgeschlagenen Schätzer werden bezüglich ihrer simulierten Risken unter quadratischer Verlustfunktion von 1000 zufällig generierten Monte Carlo Stichproben mit den Schätzern aus Singh et al. (2005) und Balasubramanian and Balakrishnan (1992) verglichen.
\end{abstract}

Keywords: Exponential Distribution, Maximum Likelihood Estimation, NonInformative Prior, Natural Conjugate Prior, Monte Carlo Simulation.

\section{Introduction}

In life testing experiments, the experimenter may not be always in a position to observe the life times of all items put on test because of time limitations and other restrictions on the data collection. Let us suppose that out of $n$ items only the first $l$ have been observed and those of the remaining $(n-l)$ components are unobserved or missing. This type of censoring is known as right type-II censoring. Another way to get censored data is to observe the largest $m$ life times. In this case the life times of the first $(n-m)$ components are missing. Such censoring is known as a left type-II censoring scheme. Moreover, if left and right censoring appears together, this is known as doubly type-II censoring. A reverse situation to doubly type-II censoring is mid censoring where the data on two extremes are available but some middle observations are censored. Furthermore, if mid censoring arises amongst the doubly censored observations, the scheme is known as a multiply type-II censoring scheme. Balakrishnan (1990) discussed a more general version of this censoring scheme where the $r_{1}$ th, $r_{2}$ th $, \ldots, r_{k}$ th, $\left(1 \leq r_{i} \leq r_{i+1} \leq n\right)$ failure times are only available. 
Under classical inference, procedures are available to deal with the complete and with certain censored samples. It is noted that under multiply type-II censoring even the likelihood estimator for a one parameter exponential distribution does not exist in closed form. Balasubramanian and Balakrishnan (1992) proposed certain approximations to solve the likelihood equations and, thus, proposed an approximate likelihood estimator for the parameter of an exponential distribution under multiply type-II censoring. For the same situation Singh et al. (2005) proposed to approximate the likelihood function to obtain an approximate likelihood estimator in closed form.

In a separate study Singh and Kumar (2005a) assumed that a point guess about the parameter is available. They proposed the use of shrinkage estimators for multiply typeII censored samples. A basic question against the use of such a shrinkage estimator is that as soon as we say that we have a point guess $\theta_{0}$ of $\theta$ with confidence $\alpha$ (known or unknown), inherently we assume that the value of $\theta$ other than $\theta_{0}$ is also possible with confidence $1-\alpha$. Thus, the very basic assumption of the shrinkage technique justifies the random character of the parameter. Moreover, summarizing all prior information in form of a single point guess has also been questioned by various authors. Alternatively it seems more reasonable to consider the problem under a Bayesian set-up. No doubt the choice of a prior distribution is our next concern. In fact, it is no serious problem, because when very little or no information on $\theta$ is priori available, the use of a non-informative prior for has been suggested by Singh and Kumar (2005b). Among the informative priors, the most widely used and perhaps the most convenient one is the natural conjugate prior. This class of priors has a number of optimal properties, most important being mathematical tractable and rich, see Martz and Waller (1982). The aim of this paper is to find the Bayes estimator for the exponential distribution using a non-informative and a natural conjugate prior for multiply type-II censored samples. It also aims to use the approximate likelihood function obtained by Singh et al. (2005) to find approximate Bayes estimators and then compare these with the exact Bayes estimators (although not available in closed form and thus evaluated only numerically).

In the next section this paper describes a Bayesian estimation procedure for the parameter of an exponential distribution based on a multiply type-II censored sample assuming a non-informative and a natural conjugate prior. The Bayes estimator has been obtained for both exact likelihood and approximate likelihood given by Singh et al. (2005). Approximate maximum likelihood estimates (MLEs) are also mentioned and compared with Bayes estimators. The Bayes estimates and likelihood estimates for an example from Lawless (1982) has been obtained in Section 3 for various values of the hyper parameters. This has been done for illustration of the proposed procedure only. Finally, the proposed estimators are compared with approximate MLEs for their risks on the basis of a Monte Carlo simulation study. A brief conclusion is given at the end.

\section{Bayes Estimation}

Consider a one parameter exponential distribution with probability density function

$$
f(x \mid \theta)=\frac{1}{\theta} \exp (-x / \theta), \quad x \geq 0, \theta>0,
$$


and cumulative distribution function

$$
F(x \mid \theta)=1-\exp (-x / \theta), \quad x \geq 0, \theta>0 .
$$

Here, $\theta$ is the expected life-time.

Suppose $n$ items, the life times of which follow an exponential distribution, are placed on test and the $r_{1}$ th, $r_{2}$ th $, \ldots, r_{k}$ th failure times are recorded. Let the $r_{i}$ th failure time $X_{r_{i}: n}$ be denoted by $Y_{i}$. Thus, $Y=\left(Y_{1}, \ldots, Y_{k}\right)$ is the multiply type-II censored sample available from (1). The likelihood function for such a sample is

$$
L(Y \mid \theta)=\frac{n !}{s ! t ! \prod_{i=1}^{k-1} u_{i}} \theta^{-k}\left(1-e^{-Y_{1} / \theta}\right)^{t} \prod_{i=1}^{k-1}\left(e^{-Y_{i} / \theta}-e^{-Y_{i+1} / \theta}\right)^{u_{i}} e^{-S_{k} / \theta}
$$

where $s=n-r_{k}, t=r_{1}-1, u_{i}=r_{i+1}-r_{i}-1$, and $S_{k}=\sum_{i=1}^{k} Y_{i}+\left(n-r_{k}\right) Y_{k}$.

\subsection{Non-Informative Prior}

A generalized non-informative prior for the parameter $\theta$ (see Martz and Waller, 1982) may be taken as

$$
g(\theta) \propto \frac{1}{\theta^{c}}, \quad c>0 .
$$

This contains Jeffery's non-informative prior as a special case when $c=1$.

The posterior distribution of $\theta$ can be obtained by combining the prior distribution and the likelihood via Bayes' theorem. This can be written as

$$
P(\theta \mid Y)=\frac{L(Y \mid \theta) g(\theta)}{\int_{0}^{\infty} L(Y \mid \theta) g(\theta) d \theta}
$$

On solving (4) with help of (2) and (3), we have

$$
P_{1}(\theta \mid Y)=\frac{\theta^{-(k+c)}\left(1-e^{-Y_{1} / \theta}\right)^{t} \prod_{i=1}^{k-1}\left(e^{-Y_{i} / \theta}-e^{-Y_{i+1} / \theta}\right)^{u_{i}} e^{-S_{k} / \theta}}{\int_{0}^{\infty} \theta^{-(k+c)}\left(1-e^{-Y_{1} / \theta}\right)^{t} \prod_{i=1}^{k-1}\left(e^{-Y_{i} / \theta}-e^{-Y_{i+1} / \theta}\right)^{u_{i}} e^{-S_{k} / \theta} d \theta}
$$

It is well known that the Bayes estimator of $\theta$ under squared error loss is the posterior mean

$$
\hat{\theta}=\int \theta P(\theta \mid Y) d \theta
$$

Substituting $P_{1}(\theta \mid Y)$ from (5) in (6), it reduce to

$$
\hat{\theta}_{1 B}=\frac{\int_{0}^{\infty} \theta^{-(k+c-1)}\left(1-e^{-Y_{1} / \theta}\right)^{t} \prod_{i=1}^{k-1}\left(e^{-Y_{i} / \theta}-e^{-Y_{i+1} / \theta}\right)^{u_{i}} e^{-S_{k} / \theta} d \theta}{\int_{0}^{\infty} \theta^{-(k+c)}\left(1-e^{-Y_{1} / \theta}\right)^{t} \prod_{i=1}^{k-1}\left(e^{-Y_{i} / \theta}-e^{-Y_{i+1} / \theta}\right)^{u_{i}} e^{-S_{k} / \theta} d \theta} .
$$

It may be noted here that equations (5) and (7) do not simplify to nice closed forms, due to the complex form of the likelihood function given in (2). Therefore, we propose to use a numerical integration method (namely the Gauss-Laguerre formula) for the evaluation of the integrals involved in these expressions. 
We have seen that neither the posterior distribution nor the Bayes estimate simplify to a closed form. However, while obtaining the MLE of $\theta$, it was noted by Singh et al. (2005) that using the approximate likelihood

$$
L(Y \mid \theta) \propto \frac{n !}{s ! t ! \prod_{i=1}^{k-1} u_{i}} \theta^{-\left(k+t+\sum_{i=1}^{k-1} u_{i}\right)} e^{-1 / \theta\left(s_{k}+\sum_{i=1}^{k-1} Y_{i} u_{i}\right)} Y_{1}^{t} \prod_{i=1}^{k-1}\left(Y_{i+1}-Y_{i}\right)^{u_{i}}
$$

instead of (2) added mathematical ease in further calculations and, in turn, provided a closed form for the approximate MLE. Tempted by this thought, we therefore propose to use (8) instead of (2) to find an approximate posterior and consequently an approximate Bayes estimator. On solving (4) with help of (5) and (8), we get

$$
P_{2}(\theta \mid Y)=\frac{\theta^{-\left(k+c+t+\sum_{i=1}^{k-1} u_{i}\right)} e^{-1 / \theta\left(s_{k}+\sum_{i=1}^{k-1} Y_{i} u_{i}\right)}}{\left(s_{k}+\sum_{i=1}^{k-1} Y_{i} u_{i}\right)^{-\left(k+c+t-1+\sum_{i=1}^{k-1} u_{i}\right)} \Gamma\left(k+c+t-1+\sum_{i=1}^{k-1} u_{i}\right)} .
$$

Substituting $P_{2}(\theta \mid Y)$ from (9) in (6), we get the approximate Bayes estimator of $\theta$ as

$$
\hat{\theta}_{1 A B}=\frac{s_{k}+\sum_{i=1}^{k-1} Y_{i} u_{i}}{k+c+t-2+\sum_{i=1}^{k-1} u_{i}} .
$$

Needless to mention that $\hat{\theta}_{1 A B}$ is non-negative and linear in the $Y_{i}$ 's.

\subsection{Natural Conjugate Prior}

A natural conjugate prior for the parameter $\theta$ of the exponential distribution is well known to be an inverted gamma prior, given as

$$
g(\theta) \propto \theta^{-(b+1)} e^{-a / \theta}, \quad a, b>0,
$$

where the hyper parameters $a$ and $b$ are chosen to reflect our beliefs. It may also be noted that (11) is a proper prior for a suitable choice of constants of proportionality provided $a>0$. For $a=0,(11)$ reduce to a general class of improper (quasi) priors. Moreover, if both $a$ and $b$ are taken equal to zero, it reduces to the well known Jeffrey's non-informative prior.

Substituting $g(\theta)$ from (11) and $L(Y \mid \theta)$ from (2), we get the posterior distribution under conjugate prior as

$$
P_{3}(\theta \mid Y)=\frac{\theta^{-(k+b+1)}\left(1-e^{-Y_{1} / \theta}\right)^{t} \prod_{i=1}^{k-1}\left(e^{-Y_{i} / \theta}-e^{-Y_{i+1} / \theta}\right)^{u_{i}} e^{-\left(a+S_{k}\right) / \theta}}{\int_{0}^{\infty} \theta^{-(k+b+1)}\left(1-e^{-Y_{1} / \theta}\right)^{t} \prod_{i=1}^{k-1}\left(e^{-Y_{i} / \theta}-e^{-Y_{i+1} / \theta}\right)^{u_{i}} e^{-\left(a+S_{k}\right) / \theta} d \theta} .
$$

Therefore, the Bayes estimator for conjugate prior can be obtained by substituting $P_{3}(\theta \mid Y)$ from (12) in (6), which reduces after simplification to

$$
\hat{\theta}_{2 B}=\frac{\int_{0}^{\infty} \theta^{-(k+b)}\left(1-e^{-Y_{1} / \theta}\right)^{t} \prod_{i=1}^{k-1}\left(e^{-Y_{i} / \theta}-e^{-Y_{i+1} / \theta}\right)^{u_{i}} e^{-\left(a+S_{k}\right) / \theta} d \theta}{\int_{0}^{\infty} \theta^{-(k+b+1)}\left(1-e^{-Y_{1} / \theta}\right)^{t} \prod_{i=1}^{k-1}\left(e^{-Y_{i} / \theta}-e^{-Y_{i+1} / \theta}\right)^{u_{i}} e^{-\left(a+S_{k}\right) / \theta} d \theta} .
$$


Once again, we see that due to the complex form of the exact likelihood, the posterior distribution and the Bayes estimator do not reduce in nice closed forms and, therefore, for solving (12) and (13), one can make use numerical of integration methods. On other hand, if one uses the approximate likelihood given in (8) instead of the exact likelihood given in (2), closed form expressions for the approximate posterior distribution and for the Bayes estimator are obtained.

To get the approximate posterior for a conjugate prior, we substitute $L(Y \mid \theta)$ from (8) and $g(\theta)$ from (11) in (4). After simplification, it reduces to

$$
P_{4}(\theta \mid Y)=\frac{\theta^{-\left(k+b+t+1+\sum_{i=1}^{k-1} u_{i}\right)} e^{-1 / \theta\left(a+s_{k}+\sum_{i=1}^{k-1} Y_{i} u_{i}\right)}}{\left(a+s_{k}+\sum_{i=1}^{k-1} Y_{i} u_{i}\right)^{-\left(k+b+t+\sum_{i=1}^{k-1} u_{i}\right)} \Gamma\left(k+b+t+\sum_{i=1}^{k-1} u_{i}\right)} .
$$

To obtain the approximate Bayes estimator for a conjugate prior under squared error loss, we substitute the approximate posterior given in (6) and, after simplification, the approximate Bayes estimator is

$$
\hat{\theta}_{2 A B}=\frac{a+s_{k}+\sum_{i=1}^{k-1} Y_{i} u_{i}}{k+b+t-1+\sum_{i=1}^{k-1} u_{i}} .
$$

$\hat{\theta}_{2 A B}$ is a linear function of the $Y_{i}$ 's and its non-negativity is obvious. The approximate MLE based on the approximate likelihood (8) as obtained by Singh et al. (2005) is

$$
\hat{\theta}_{U A}=\frac{s_{k}+\sum_{i=1}^{k-1} Y_{i} u_{i}}{k+t+\sum_{i=1}^{k-1} u_{i}} .
$$

The approximate MLE of $\theta$ as proposed by Balasubramanian and Balakrishnan (1992) is

$$
\hat{\theta}_{B L}=\frac{\sum_{i=0}^{k-1}\left(\delta_{i} Y_{i}+\left(1-\delta_{i}\right) Y_{i+1}\right) u_{i}+S_{k}}{k-\sum_{i=0}^{k-1} u_{i} \gamma_{i}},
$$

where $r_{0}=0, Y_{0}=0, p_{i}=r_{i} /(n+1), q_{i}=1-p_{i}, \delta_{i}=q_{i} /\left(q_{i}-q_{i+1}\right)-\left(q_{i} q_{i+1}\right) /\left(q_{i}-\right.$ $\left.q_{i+1}\right)^{2} \log \left(q_{i} /\left(q_{i+1}\right)\right)$ and $\gamma_{i}=\left(q_{i+1} \log q_{i+1}-q_{i} \log q_{i}\right) /\left(q_{i}-q_{i+1}\right)+\delta_{i} \log q_{i}+(1-$ $\left.\delta_{i}\right) \log q_{i+1}$, for $i=0,1, \ldots, k-1$. It may be noted that $\hat{\theta}_{U A}, \hat{\theta}_{B L}, \hat{\theta}_{1 A B}$, and $\hat{\theta}_{2 A B}$ are linear functions of the $Y_{i}$ 's and the non-negativity of all estimators is evident.

\section{Illustrative Example}

Consider the following multiply type-II censored data, which represents failure times in minutes for a specific type of electrical insulation in an experiment in which the insulation was subjected to a continuously increasing voltage stress (see Lawless, 1982, pp.138):

$$
12.3,21.8,-, 28.6,43.2,46.9,-, 75.3,95.5,98.1,138.6,-
$$

Here, twelve items were placed on a life-testing experiment and the third and seventh observations are censored since the experimenter fail to observe their failure times. The last observation is also censored since the experimentation was stopped as soon as the eleventh failure occurred. 
Table 1: MLEs and Bayes estimates using a non-informative prior

\begin{tabular}{ccccc}
\hline$c$ & $\hat{\theta}_{B L}$ & $\hat{\theta}_{U A}$ & $\hat{\theta}_{1 B}$ & $\hat{\theta}_{1 A B}$ \\
\hline 1 & 71.3462 & 69.7818 & 76.76 & 81.0454 \\
2 & 71.3462 & 69.7818 & 69.7818 & 72.2881 \\
3 & 71.3462 & 69.7818 & 63.9667 & 65.5515 \\
4 & 71.3462 & 69.7818 & 59.0462 & 60.0841 \\
5 & 71.3462 & 69.7818 & 54.8286 & 55.5301 \\
6 & 71.3462 & 69.7818 & 51.1733 & 51.6995 \\
\hline
\end{tabular}

Table 2: MLEs and Bayes estimates using a natural conjugate prior

\begin{tabular}{cccccc}
\hline$a$ & $b$ & $\hat{\theta}_{B L}$ & $\hat{\theta}_{U A}$ & $\hat{\theta}_{2 B}$ & $\hat{\theta}_{2 A B}$ \\
\hline & 1 & 71.3462 & 69.7818 & 69.8727 & 72.3791 \\
& 2 & 71.3462 & 69.7818 & 64.05 & 65.6343 \\
& 3 & 71.3462 & 69.7818 & 59.1231 & 60.1603 \\
1 & 4 & 71.3462 & 69.7818 & 54.9 & 55.6007 \\
& 5 & 71.3462 & 69.7818 & 51.24 & 51.7655 \\
& 6 & 71.3462 & 69.7818 & 48.0375 & 48.5247 \\
\hline & 1 & 71.3462 & 69.7818 & 69.9636 & 72.4702 \\
& 2 & 71.3462 & 69.7818 & 64.1333 & 65.7172 \\
& 3 & 71.3462 & 69.7818 & 59.2 & 60.2364 \\
2 & 4 & 71.3462 & 69.7818 & 54.9714 & 55.6713 \\
& 5 & 71.3462 & 69.7818 & 51.3067 & 51.8315 \\
& 6 & 71.3462 & 69.7818 & 48.1 & 48.5869 \\
\hline & 1 & 71.3462 & 69.7818 & 70.1455 & 72.6523 \\
& 2 & 71.3462 & 69.7818 & 64.3 & 65.8829 \\
& 3 & 71.3462 & 69.7818 & 59.3538 & 60.3887 \\
4 & 4 & 71.3462 & 69.7818 & 55.1143 & 55.8124 \\
& 5 & 71.3462 & 69.7818 & 51.44 & 51.9635 \\
& 6 & 71.3462 & 69.7818 & 48.225 & 48.7112 \\
\hline
\end{tabular}

Based on this sample, we calculated the exact Bayes estimator $\hat{\theta}_{1 B}$ given in (7) using Gauss-Laguerre quadrature formula for various values of the hyper parameters. The approximate Bayes estimator $\hat{\theta}_{1 A B}$ given in (10) has also been calculated. The estimates are shown in Table 1 along with the approximate likelihood estimates discussed by Singh et al. (2005).

For the multiply type-II censored sample mentioned above and considering a conjugate prior, we calculated the exact Bayes estimates $\hat{\theta}_{2 B}$ given in (13) using GaussLaguerre quadrature for various values of the hyper parameters. The approximate Bayes estimator $\hat{\theta}_{2 A B}$ given in (14) has also been calculated for the same values of the hyper parameters. The results have been summarized in Table 2 along with the approximate likelihood estimates as given by Singh et al. (2005).

However, on the basis of a single sample, one should not conclude about the proper choice of hyper parameters to be used for the estimates. And, therefore, for the complete study of the performances of these estimators, we propose to study the estimators for their long run use on the basis of their risks (expected loss over whole sample space) and the same is given in the next section. 


\section{Monte Carlo Simulation Study}

It may be noted from the previous section that the approximate Bayes estimators $\hat{\theta}_{1 A B}$ and $\hat{\theta}_{2 A B}$ are in closed forms and their risks can easily be calculated. The exact Bayes estimators $\hat{\theta}_{1 B}$ and $\hat{\theta}_{2 B}$ involve integral expressions and their risks can not be obtained in closed forms. Therefore, we propose to study the behavior of the estimators on the basis of their simulated risks. For this purpose, a Monte Carlo study of 1000 samples each of sizes 10,20 and 30 was conducted for $\theta=0.5(0.5) 10$. To cover different censoring fractions, we assigned different values to $k$ and to the $r_{i}$ 's (e.g., for $k=3, r_{i}$ is assigned to 1,2,3; $1,3,5 ; 6,7,8$; and $2,4,7$, respectively, whereas for $k=5, r_{i}$ is chosen as $1,2,5,6,8$; $3,4,7,8,9 ; 1,2,3,4,5$; and $6,7,8,9,10$, respectively, when $n=10$ ).

\subsection{Comparison of Risks Using a Non-Informative Prior}

The simulated risks under squared error loss for the Bayes estimator $\hat{\theta}_{1 B}$ and $\hat{\theta}_{1 A B}$ have been obtained for a number of values of the hyper parameter $c$. The values considered for $c$ are $1,2,3,4,5,6$. The curves for the risk of the estimators were traced for the variation in the values of $c$ keeping $n, \theta, k$, and $r_{i}$ fixed. It was noted that the shapes of the risks curves remains more or less same for the variation in the values of $n$ and $\theta$. Therefore the curves of risks are only partially shown in Figure 1 for $n=10, \theta=5$, and $r_{i}$ assigned to $1,2,3,4,5 ; 1,2,5,6,8 ; 3,4,7,8,9 ;$ and $6,7,8,9,10$, respectively. It may be noted from these figures that if early failures constitute the observed values, for a wide range of values of $c$, the Bayes estimator has smaller risks than MLEs. As more and more high order failures are included in the observed values (keeping the total number of observations fixed), this range of $c$ decreases. It is noted from an extensive study for various choice of $n, \theta$, and $r_{i}$ that most often the Bayes estimators have smallest risk if $c$ is close to 3 for $\hat{\theta}_{1 A B}$ and 2 for $\hat{\theta}_{1 B}$. It is also noted from the results that the risks of Bayes estimators are less than those of the approximate MLEs if early failures are observed. Contrary to it, if only high order failures are observed, there may not be any gain or may be marginal gain in the sense of reduction in risk due to the use of Bayes estimators even with a proper choice of hyper parameter $c$. It may be interesting to note here that if high order failures constitute the observations, the approximate Bayes estimator has smaller risk than that of the exact Bayes estimator. On the other hand, if early failures are included in the observations, the exact Bayes estimator has smaller risk for small values of $c$ whereas for large values of $c$, the approximate Bayes estimator has smaller risk.

The curves for the risk of the estimators were also traced for variation in the values of $\theta$ keeping the other parameters fixed. It is noted from the figures that the relative positions of the risk curves for various estimators remain the same for variation in $n$, although the risks decrease in magnitude as $n$ increases. However, the type of observation (early failures or high order failures) in the sample effects the relative position of the risk curves more than the number of observations. It has also been noted above that the choice of $c$ does effect the behavior of risks but the values of $c$ close to 3 provide smaller risk for Bayes estimators and for large and small values of $c$, the risk of Bayes estimators become larger than the risk of approximate MLEs. Therefore, in Figure 2 we have shown the risk of the estimators only for $n=10, c=1,3,6 ; \theta=2,5,10 ; k=5$, and $r_{i}$ set 

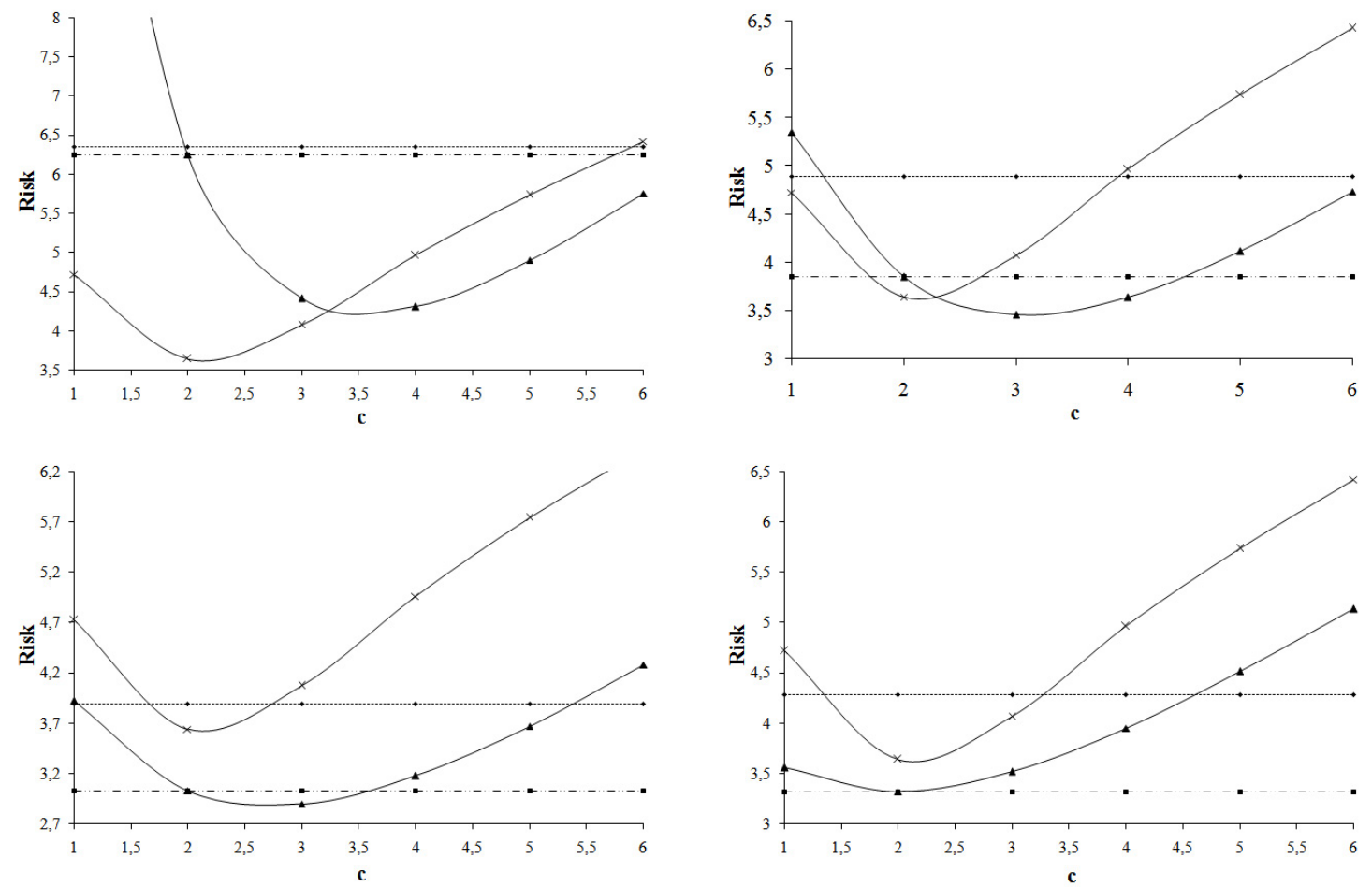

Figure 1: Risk of estimators for $n=10, \theta=5$, if $r_{i}=1,2,3,4,5$ or $1,2,5,6,8$ (above), and $r_{i}=3,4,7,8,9$ or $6,7,8,9,10$ (below). $\hat{\theta}_{B L}$ and $\hat{\theta}_{U A}$ shown as dotted and dashed lines; $\hat{\theta}_{1 A B}$ and $\hat{\theta}_{1 B}$ as solid lines with triangle and box symbols, respectively.
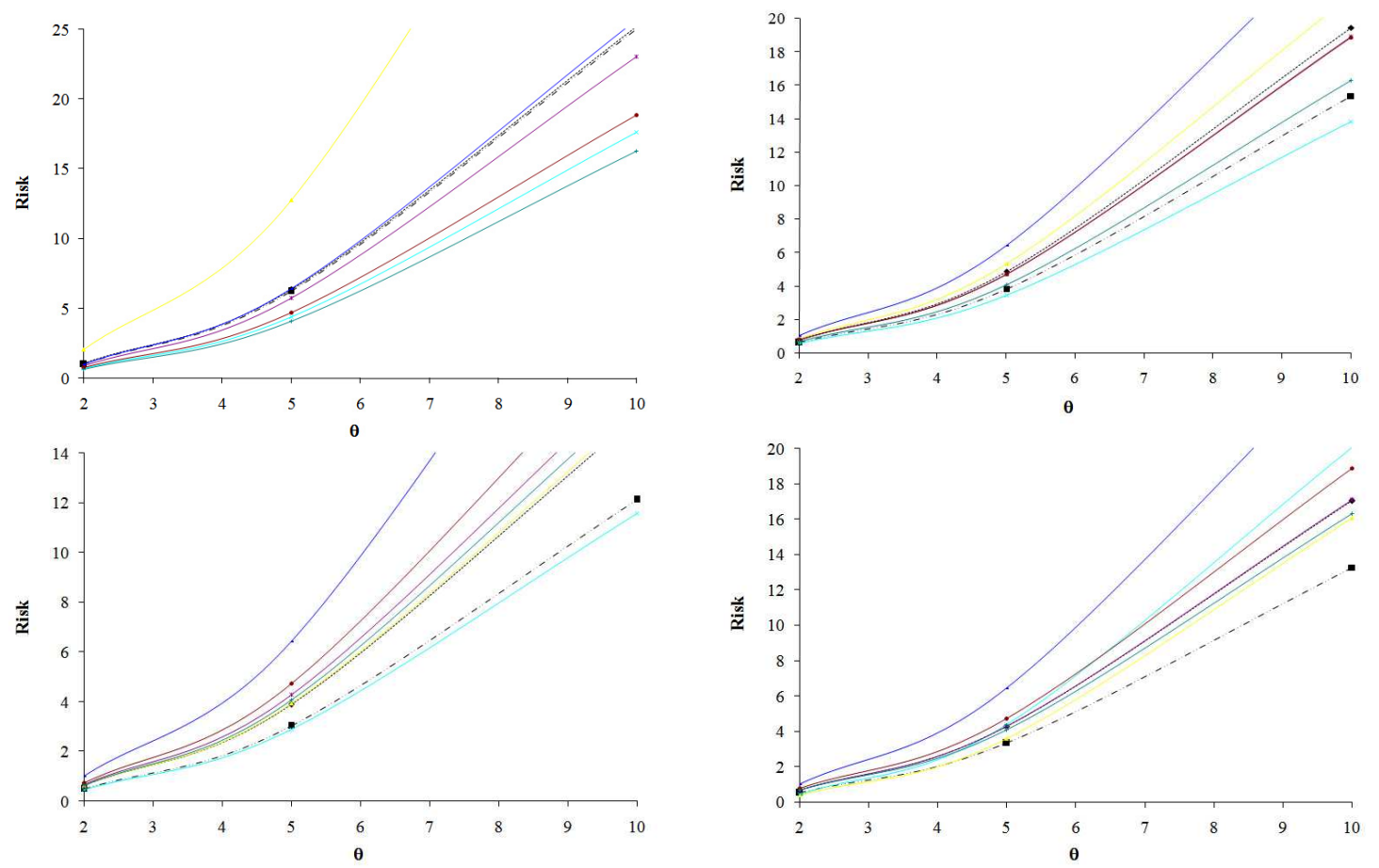

Figure 2: Risk of estimators for $n=10$, if $r_{i}=1,2,3,4,5$ or $1,2,5,6,8$ (above), and $r_{i}=3,4,7,8,9$ or $6,7,8,9,10$ (below). 
as before. However, the following discussion is based on the extensive simulation study as mentioned in the beginning of this section. An obvious finding was noted by us that as $\theta$ increases the risk of all the estimators increase but the rate of increase varies from estimator to estimator. It is further noted that the risk of the approximate Bayes estimate with $c=3$ is often quite close the risk of the approximate MLE $\hat{\theta}_{U A}$. If more early failures are observed in the sample, the risk of the approximate Bayes estimate is slightly smaller than the risk of $\hat{\theta}_{U A}$. However, if high order failures are observed the situation is reversed. It is also noted that if more early failures constitute the observations, the exact Bayes estimate with $c=2$ has smaller risk than those of the approximate Bayes estimator and the approximate MLE. But this trend is changed if some or more of high order failures were taken in the observations, keeping the total number of observations fixed.

\subsection{Comparison of Risks Using a Natural Conjugate Prior}

It is obvious that the risk of Bayes estimators using a conjugate prior will depend on the choice of the hyper parameters $a$ and $b$ in addition to the parameters $n, k$, and $r_{i}$. The risk of the estimators has been simulated for various choices of the parameters. The values considered for simulating the risks are $0.5,1(1) 8.0$ for both $a$ and $b$. It is noted from the results that a change in the values of $n$ and $k$ does not effect the relative position of the risk curves for fixed values of other parameters and hyper parameters, although the risk, in general, decreases as $n$ and $k$ increase. Therefore, the curves have been shown for $n=10$ and $k=5$ only. As seen in the previous subsection the relative position of the risk curves is affected by the type of observations (early or high order failures) included in the observed sample. Thus, we have considered $r_{i}$ as before, for showing the behavior of the risk curves. Figure 3 show the variation in the risks with respect to a change in the value of $\theta=2,5,10$ for fixed values of the other parameters. Here $a$ and $b$ both are set to 4. It can be seen that as $\theta$ increases the risk of all estimators increases. For $a=b=4$ the risk of $\hat{\theta}_{2 A B}$ is smaller than that of $\hat{\theta}_{2 B}$ in almost all cases. It is also seen that if high order failures are included, the risk of all estimators decreases but the rate of decrease varies from estimator to estimator. A faster decrease is noticed for the approximate MLE than for the Bayes estimators. Among the Bayes estimators the rate of decrease is larger for $\hat{\theta}_{2 A B}$ than for $\hat{\theta}_{2 B}$. If most of the observations are high order failure times, $\hat{\theta}_{U A}$ has risk either close to or smaller than the risk of $\hat{\theta}_{2 A B}$.

To study the effect of the hyper parameters $a$ and $b$ on the risks, we traced the risk for various values of a hyper parameter keeping the other parameters fixed. For variation in the values of $b$, the risk curves are shown partially in Figure 4. The values of $n, \theta$, and $a$ are fixed arbitrarily at 10,5, and 4, respectively, and $b=0.5(1) 5$. It may be seen from these figures that as $b$ increases, the risk of Bayes estimators decrease first, attains minima and then increase. It may also be seen that except for small values of $b$, the risk of the exact Bayes estimator is always larger than that of the approximate Bayes estimator, although for large values of $b$, the risk of the approximate Bayes estimator becomes greater than the risk of $\hat{\theta}_{U A}$. On the other hand, to study the effect of the hyper parameter $a$ on the risk of Bayes estimators, we traced the risk for various values of $a$ keeping the other parameters fixed. Such risk curves are partially summarized in Figure 5 for $n=10, b=4$, and $\theta=5$. It is noted from the risk curves that in the considered range of variation of $a$ (i.e., 1 to 10) 

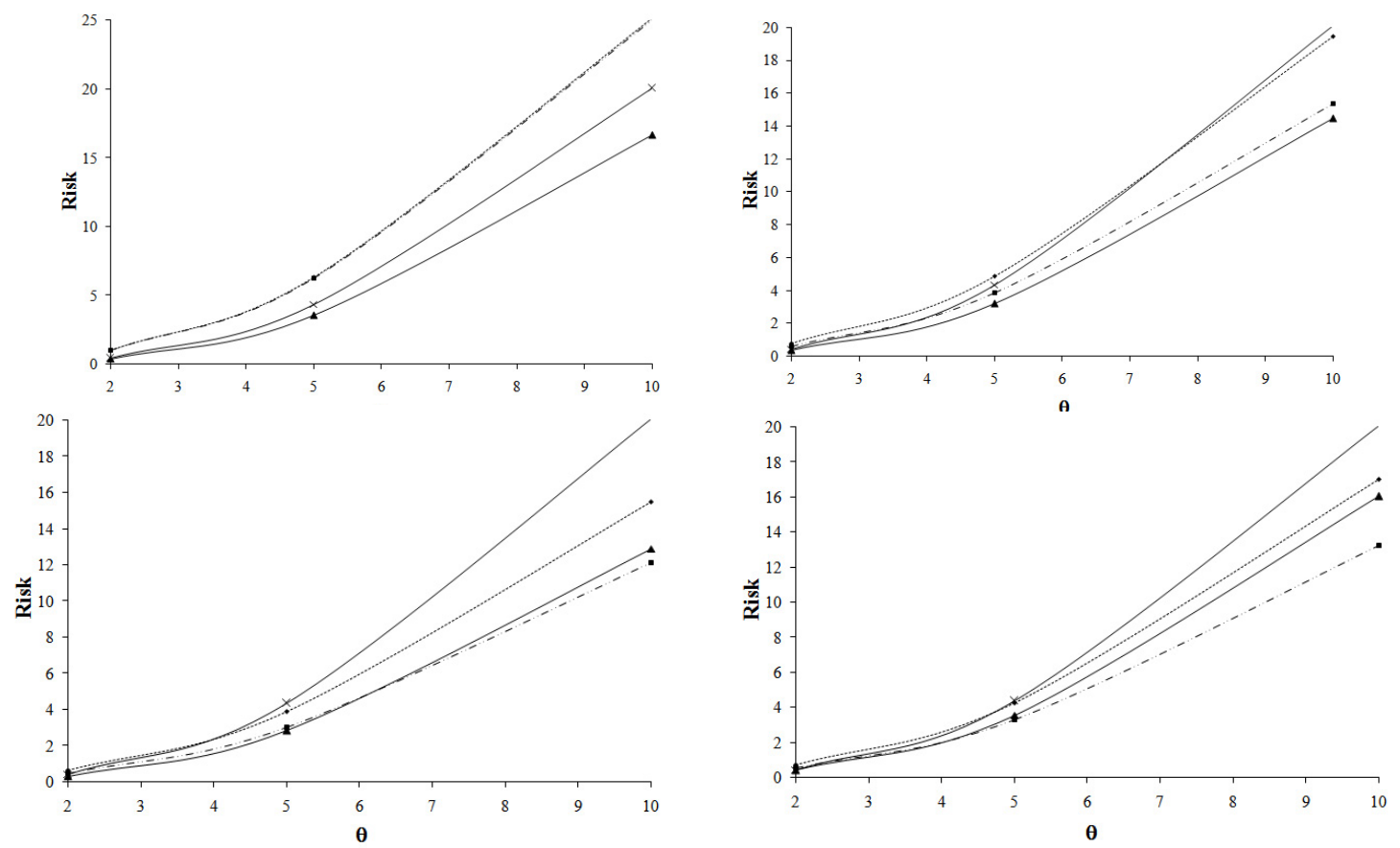

Figure 3: Risk of estimators for $n=10, a=b=4$, if $r_{i}=1,2,3,4,5$ or $1,2,5,6,8$ (above), and $r_{i}=3,4,7,8,9$ or $6,7,8,9,10$ (below). $\hat{\theta}_{B L}$ and $\hat{\theta}_{U A}$ shown as dotted and dashed lines; $\hat{\theta}_{2 A B}$ and $\hat{\theta}_{2 B}$ as solid lines with triangle and box symbols, respectively.
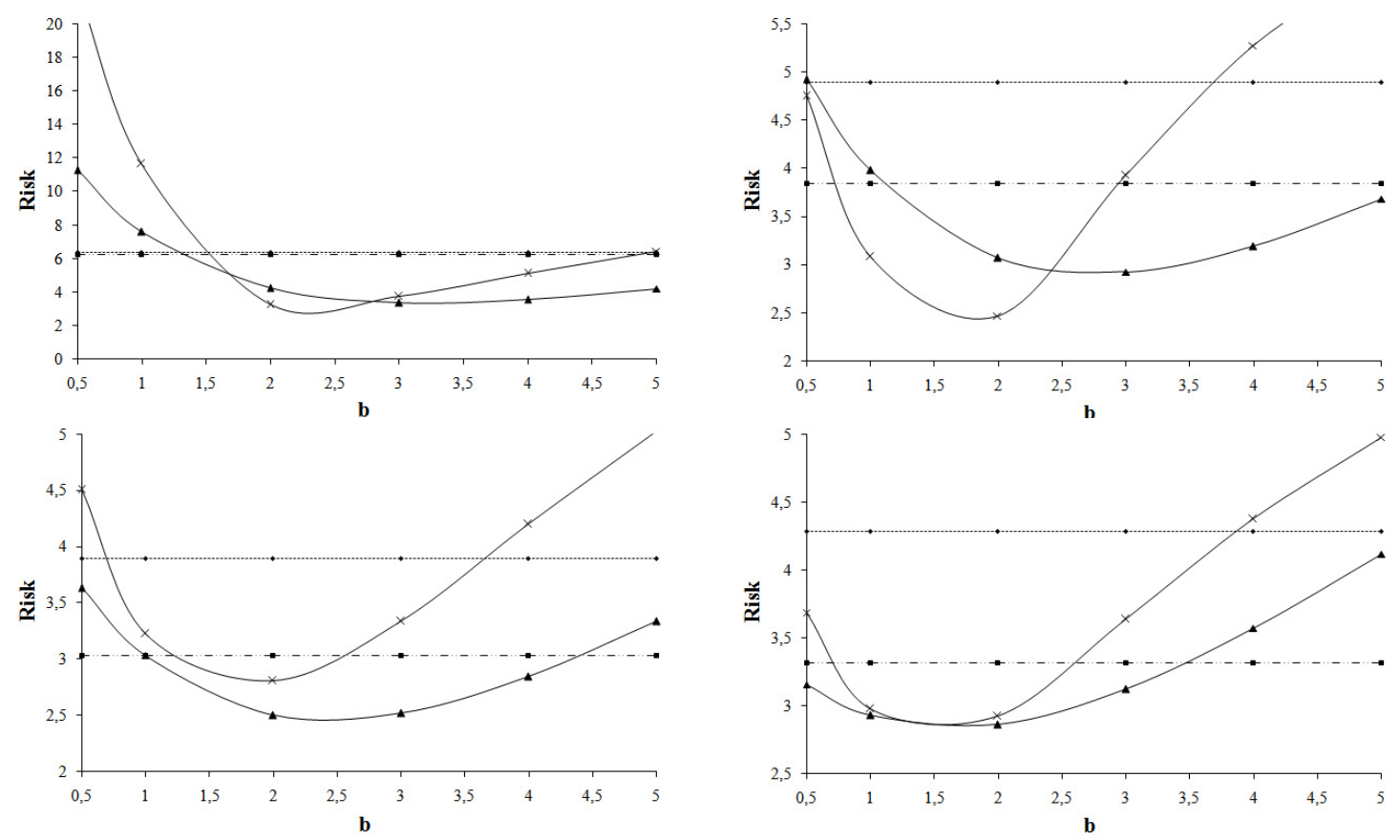

Figure 4: Risk of estimators for $n=10, a=4, \theta=5$, if $r_{i}=1,2,3,4,5$ or $1,2,5,6,8$ (above), and $r_{i}=3,4,7,8,9$ or $6,7,8,9,10$ (below). $\hat{\theta}_{B L}$ and $\hat{\theta}_{U A}$ shown as dotted and dashed lines; $\hat{\theta}_{2 A B}$ and $\hat{\theta}_{2 B}$ as solid lines with triangle and box symbols, respectively. 

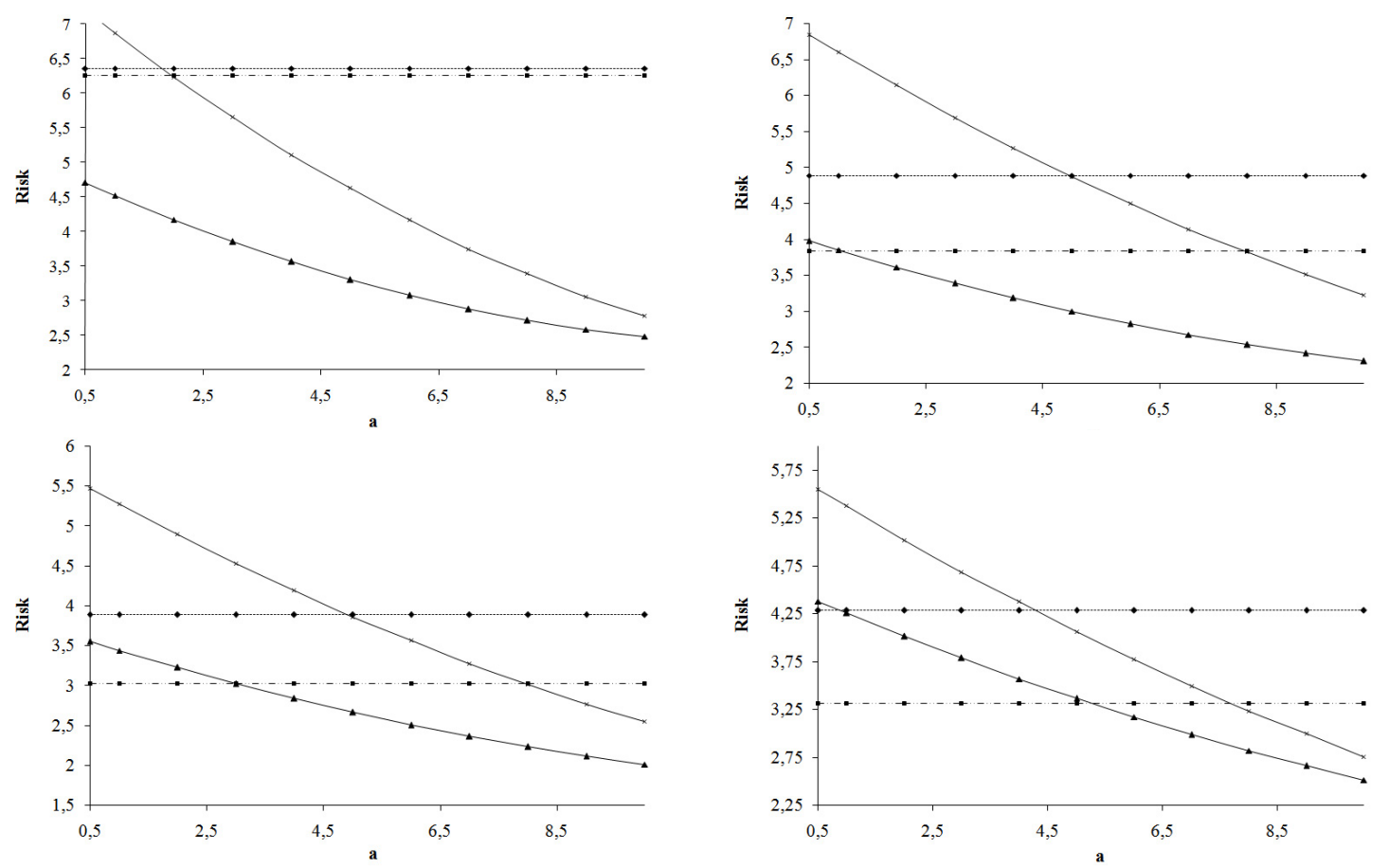

Figure 5: Risk of estimators for $n=10, b=4, \theta=5$, if $r_{i}=1,2,3,4,5$ or $1,2,5,6,8$ (above), and $r_{i}=3,4,7,8,9$ or $6,7,8,9,10$ (below). $\hat{\theta}_{B L}$ and $\hat{\theta}_{U A}$ shown as dotted and dashed lines; $\hat{\theta}_{2 A B}$ and $\hat{\theta}_{2 B}$ as solid lines with triangle and box symbols, respectively.

the risk of the Bayes estimators decreases as $a$ increases, though the rate of decrease in the risk is more when the sample consists of more early failure times. It may be worth to mention here that the decreasing trend of the risk of Bayes estimator continues up to moderately larger values of $a$. However, if $a$ further increases (quite often greater than 20), the risk of the Bayes estimators start increasing. It is also noted from the detailed study that the value of $a$, from where the decreasing trend of risk of Bayes estimators changes to increasing trend, is smaller when the observed sample values include more early failure times than those situations where sample observation contain more of higher order failure times. It may be worth to mention that if $\theta$ is small the risks of all estimators are small in the magnitude but for small choices of the values of $a$ and $b$, the risk of the Bayes estimators can be made smaller than that of the approximate MLE. On the other hand, if $\theta$ is moderate or large, a moderate value of $1<b<3$ and for a wide range of large values of $5<a<20$, the risk of the approximate Bayes estimator is smaller than the risk of the other estimators.

\section{Conclusion}

From the above discussion it is clear that under the classical criterion of the comparison (i.e., risk defined as the average loss over whole sample space), the Bayes estimators proposed here provide a flexible estimator in the sense that by a proper choice of the hyper parameter, the Bayes estimators may have smaller risk than the approximate MLE. If one is interested in using only the sample information, then $\hat{\theta}_{1 A B}$ with $c=3$ is recommended to be used because it is based on a non-informative prior and provides small risk. This recommendation is valid only if the sample does not contain mostly higher ordered failure 
times. If sample observations are left type-II censored, the use of $\hat{\theta}_{U A}$ is recommended. It is also mentioned here that the Bayes estimator for conjugate prior offers a larger flexibility. It is observed that if $\theta$ is small, small values of $a$ and $b$ provide a reduction in risk due to the use of the Bayes estimators whereas if $\theta$ is large, moderate value of $a$ and $b$ may be required. The risk of the approximate Bayes estimate, however, improves for a wide range of $a$ and $b$ between 2 and 3, particularly when the sample includes mainly early failures. Therefore, one can recommend to use $\hat{\theta}_{2 A B}$ with $2<b<3$ and $6<a<14$. If the sample includes early failures and $\theta$ is not small, comparatively small values of $a$ are going to be profitable. However, if sample includes higher order failure times, approximate Bayes estimates with large $a$ and a moderate value of $b$ can be used or in such situation use of $\hat{\theta}_{U A}$ can be also recommended.

\section{Acknowledgement}

We are highly thankful to the editor and referees for their valuable suggestions without which the paper could not have taken its present form.

\section{References}

Balakrishnan, N. (1990). On the maximum likelihood estimation of the parameters of exponential distribution based on multiply-II censored sample. Journal of Applied Statistics, 17, 55-61.

Balasubramanian, K., and Balakrishnan, N. (1992). Estimation for one and two parameter exponential distribution under multiply-II censoring. Statistische Hefte, 33, 203216.

Lawless, J. F. (1982). Statistical Models \& Methods for Lifetime Data. New York: John Wiley and Sons.

Martz, H. F., and Waller, R. A. (1982). Bayesian Reliability Analysis. New York: John Wiley and Sons.

Singh, U., and Kumar, A. (2005a). Shrinkage estimators for exponential scale parameter under multiply type II censoring. Austrian Journal of Statistics, 34, 39-49.

Singh, U., and Kumar, A. (2005b). Bayes estimator for one parameter exponential distribution under multiply-II censoring. Indian Journal of Mathematics amd Mathematical Sciences, 1, 23-33.

Singh, U., Kumar, A., and Upadhyay, S. K. (2005). Maximum likelihood estimators of location and scale parameters of the exponential distribution under multiply-II censoring. Assam Statistical Review, 19, 30-43.

Authors' addresses:

Umesh Singh

Department of Statistics

Banaras Hindu University

Varanasi

India

\author{
Anil Kumar \\ Department of Statistics \\ Faculty of Science and Technology \\ Mahatma Gandhi Kashi Vidya Peeth \\ Varanasi-221 005 , \\ India \\ E-mail: dranilkrbhu@yahoo.co.in
}

